\title{
Turbulent Spot in Linearly Stable Taylor Couette Flow
}

\author{
Arjang Alidai ${ }^{1,2}$ - Arnoud J. Greidanus ${ }^{1}$. René Delfos ${ }^{1}$. \\ Jerry Westerweel ${ }^{1}$
}

Received: 12 March 2015 / Accepted: 2 October 2015 / Published online: 14 October 2015

(C) Springer Science+Business Media Dordrecht 2015

\begin{abstract}
The transition from the laminar to the turbulent regime in linearly stable shear flows, for example, pipe and plane Couette flows, occurs abruptly with no precursor. The evolution of turbulent spots has been studied to better understand the dynamics of this transition and the onset of turbulence. These studies have mostly focused on pipe flows for which a finite lifetime of spots was proven. The same conclusion was drawn in the only available study performed in a Taylor Couette setup. Here, the spot lifetime is measured in a different size TC setup. It is shown that the lifetime is indeed finite and also very sensitive to boundary conditions, but not much to perturbation mechanisms. A scaling approach is provided which suggests in addition to the Reynolds number, the aspect and radius ratios are influential parameters on the lifetime. It is found that the spot size varies during its lifetime and increases with the Reynolds number that confirms the rise in turbulence proliferation by approaching the transitional point.
\end{abstract}

Keywords Transient turbulence - Taylor Couette - Turbulent spot - Local and global perturbations

Arjang Alidai

arjang.alidai@gmail.com

Arnoud J. Greidanus

a.j.greidanus@tudelft.nl

René Delfos

r.delfos@tudelft.nl

Jerry Westerweel

j.westerweel@tudelft.nl

1 Laboratory for Aero \& Hydrodynamics, Delft University of Technology, Mekelweg 2, 2628 CD

Delft, The Netherlands

2 Department of Industrial Hydrodynamics, Deltares, P.O. Box 177, 2600 MH Delft, The Netherlands 


\section{Introduction}

Despite all recent theoretical, experimental, and numerical developments in the field of fluid dynamics, the solution to the one million dollar Navier-Stokes equation has not been achieved yet; mathematically it is not even clear, if a three dimensional solution to the equation always exists and if so, if it contains no singularities [6]. Consequently, some basic aspects of flow like the onset of turbulence and its development are not completely understood. Turbulence significantly influences fluid mixing, heat transfer, kinetic energy loss, and other flow phenomena; thus its control is very beneficial. For example, by delaying turbulence in pipe flows, one can avoid considerable energy losses.

The transition from the laminar to the turbulent regime was first studied by Osborne Reynolds [18] who showed that the general character of the motion of fluids changes when Reynolds number, $R e$, reaches a critical value, $R e_{c r}$. Despite his continuing effort, he was not able to find a definite $R e_{c r}$. His last estimates were about $R e_{c r}=1900,2000$. At these $R e$ turbulence is intermittent, appearing in the form of streamwise localized structures (also called puff, spot, and patch) traveling in the downstream direction. Since then, the onset of turbulence has been numerously investigated in shear flows.

A breakthrough was made when Taylor predicted the primary flow instability from the laminar flow by applying linear stability analysis ${ }^{1}$ to the Navier-Stokes equation [22]. In flows that are linearly unstable, the transition to turbulence is straightforward; the laminar regime becomes unstable by increasing driving force and more complex flow modes form until, after a series of sequential bifurcations, turbulence sets in $[7,8,14]$. Bénard cells and Taylor vortices are well-known examples of these instabilities in the route to turbulence in such flows.

Pipe and plane Couette flows are linearly stable at all Reynolds numbers $[15,20]$. In these flows, the transition occurs abruptly at a certain $R e$ by introducing sufficiently large finite-amplitude perturbations. Wygnanski et al. [24] examined the spatial evolution of puffs triggered by controlled localized perturbation. After formation, a puff is swept downstream with the surrounding laminar flow and temporarily grows until this oversized puff splits thereby generates a new puff and increases the turbulent fraction of the flow. Puff spitting was identified as the mechanism through which turbulence spreads by spatial proliferation and was chosen to predict $R e_{c r}[16,24]$.

Although Wygnanski et al. captured the intermittent character of turbulence, they were not aware that its temporal evolution is a stochastic process. A statistical approach was first utilized by Bottin and his collaborators $[4,5]$ to investigate the flow dynamics in the transitional regime. They measured the lifetime of a turbulent spot in plane Couette flow and repeated the procedure one hundred times. They found an exponential distribution of the lifetimes. All ensuing studies agreed on [3, 11-13, 17, 23]:

- $\quad$ statistical approach to the transitional regime,

- exponential probability distribution of spot lifetime,

- increase in characteristic mean lifetimes with $R e$.

However, they differed in their conclusions with respect to the lifetime growth with $R e$. While some inferred from the rapid increase of mean lifetimes that the lifetime diverges at $R e_{c r}$, and thus turbulence sustains [4, 5, 11, 17, 23], others reported a bounded lifetime and concluded a transient nature for turbulence $[12,13]$. This dispute was finally settled by

\footnotetext{
${ }^{1}$ With the linear stability analysis a certain (flow) state is investigated under the influence of infinitesimal perturbations. Depending on $R e$ and initial flow state, these perturbations can either grow exponentially in time leading to the change of flow state or they decay.
} 
Avila et al. [2] whose extensive numerical investigation proved a super-exponential scaling of mean lifetimes that indicates no threshold of divergence exists.

Bounded lifetime of spots does not solely mean that turbulence is a transient state at all Reynolds numbers. First comparison of the purely temporal behavior of spots, which is reflected in the lifetimes, with their spatial dynamics (i.e. the spreading of turbulence in space) was conducted by Moxey \& Barkley [16]. Based on their extensive numerical investigation, they postulated that although any individual spot has a finite probability of decay, turbulence has a finite probability of sustaining indefinitely. This was explained by the fact that the spread of turbulence is sufficiently faster than the decay of spots at $R e_{c r}$. At the moment, no experimental data that support this postulation is available. Pipe and plane Couette setups are prone to entry disturbances and do not allow for long measurement times owing to their limited length (the lifetime of spots become astronomically long for high $R e$ ). Taylor Couette setup, which has a closed geometry, is more suitable to investigate the temporal and spatial behavior of spots. In case of a stationary inner cylinder, the TC flow is linearly stable for all $R e$ [10], and thus its results can be extended to pipe and plane Couette flows.

There is only one available study of the spot lifetime in a TC setup [3] in which a finite lifetime was reported. However, no details about the effects of parameters such as perturbation mechanisms (global or local), geometrical properties, and boundary conditions were provided. In the current work, the spot lifetime is reexamined. Influential parameters on the lifetime are specified and their impacts are investigated. Considering their effects, a scaling approach is suggested which results in a more general representation of the lifetime in TC flows. The spot size is also measured at several $R e$ to investigate its spatial evolution.

\section{Materials and Methods}

\subsection{Geometry of setup}

The experimental apparatus (as shown in Fig. 1) consisted of two vertical concentric cylinders made of plexiglass with the inner and outer radii of $r_{i}=110 \pm 0.05 \mathrm{~mm}$ and

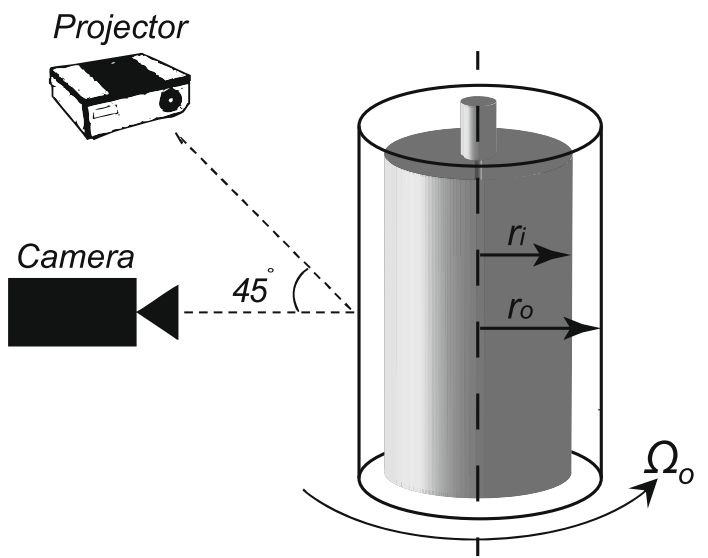

(a)

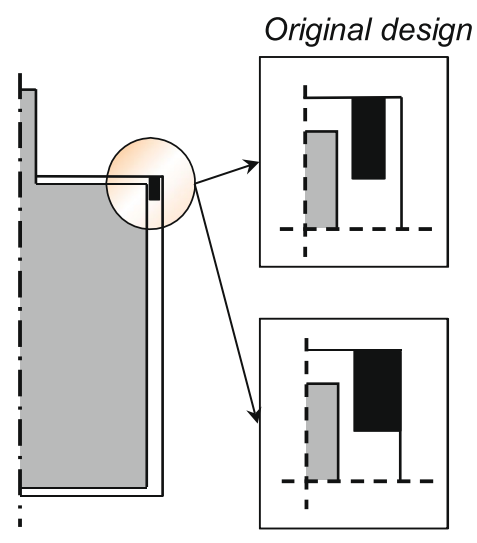

Improved design

Fig. 1 Schematic picture of setup, a perspective view and $\mathbf{b}$ cross section view 
Table 1 Geometrical properties of setup

\begin{tabular}{lllllll}
\hline Parameter & $r_{i}(\mathrm{~mm})$ & $r_{o}(\mathrm{~mm})$ & $d(\mathrm{~mm})$ & $\eta$ & $\Gamma$ & $R e_{c r}$ \\
\hline Current study & 110 & 120 & 10.0 & 0.917 & 22 & $\approx 6000$ \\
Borrero-Echeverry et al. [3] & 66.38 & 76.20 & 9.82 & 0.871 & 36 & $\approx 16000$ \\
\hline
\end{tabular}

$r_{o}=120 \pm 0.05 \mathrm{~mm}$ (thus a gap size of $d=10 \mathrm{~mm}$ ). Both cylinders were connected to two independent Brushless DC motors (Maxon, 250W) and a maximum frequency of $10 \mathrm{~Hz}$ with an absolute precision of $\pm 0.01 \mathrm{~Hz}$. The outer cylinder was attached to the top and bottom plates with a gap of $1.5 \mathrm{~mm}$ between the end surfaces of the inner cylinder and the plates. The height of the inner cylinder was $L=220 \mathrm{~mm}$. Two non-dimensional numbers are defined (as summarised in Table 1): the aspect ratio $\Gamma=L / d$ and the radius ratio $\eta=r_{i} / r_{o}$. Consistent with the previous study [3], the Reynolds number is described as $R e=r_{o} \Omega_{o} d / v$, with $\Omega_{o}$ and $v$ as the angular velocity of the outer cylinder and the kinematic viscosity of fluid respectively.

\subsection{Perturbation mechanism}

In this setup, the abrupt transition occurred at $R e_{c r} \sim 6000^{2}$, and hence, lifetime measurements were conducted for Reynolds numbers below this value. First, laminar flow was generated by slowly accelerating the outer cylinder up to a certain frequency (corresponding to the desired Reynolds number) while the inner cylinder was at rest. After reaching this frequency, the system was allowed to run for $100 \mathrm{sec}$. In order to create a turbulent spot, two different mechanisms were deployed: Global Perturbation and Local Perturbation (hereafter GP and LP). GP was introduced by sudden counter rotation of the inner cylinder (at a frequency of $0.65 \mathrm{~Hz}$ ) for $3 \mathrm{sec}$. As mentioned in Section 1, as long as a spot is created, its lifetime does not depend on the strength or duration of perturbation. Thus, in all experiments, the flow was disturbed by the same frequency. LP was imposed on the mean flow by simultaneous injection and suction of water (in the radial direction) for $1 \mathrm{sec}$ through two $0.9 \mathrm{~mm}$ holes located on the half height of the inner cylinder.

\subsection{Flow visualisation and image processing}

Distilled water was used as working fluid with a viscosity of $v=1.004 \times 10^{-6} \mathrm{~m}^{2} / \mathrm{s}$ (at a temperature of $T=20{ }^{\circ} \mathrm{C}$ ). To visualise flow patterns, Iriodin pigments (Iriodin 121) were mixed with water at a concentration of $0.7 \%$ volume (the viscosity of the mixture was measured and found to be the same as that of water). When these pigments are put into motion, they are aligned with their larger dimensions parallel to the planes of shear (similar

\footnotetext{
${ }^{2} R e_{c r}$ in the setup was measured as follow: the laminar flow was generated at the outer cylinder frequency of $0.53 \mathrm{~Hz}$ (corresponding to $R e=4000$ ) while the inner cylinder was at rest. The system was allowed to run for $100 \sec \left(\sim 1\right.$ radial diffusion times $\left.t_{d}=d^{2} / v\right)$ to rule out any disturbance. If the flow became turbulent after this time, the Reynolds number was considered as the critical one, otherwise, the frequency of the outer cylinder was increased by $0.01 \mathrm{~Hz}$, and the flow was checked again. This procedure was repeated till the transition to turbulence took place.
} 


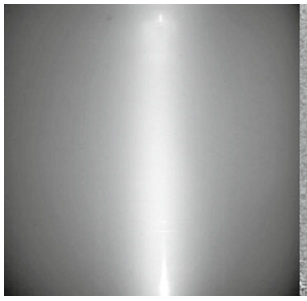

(a)

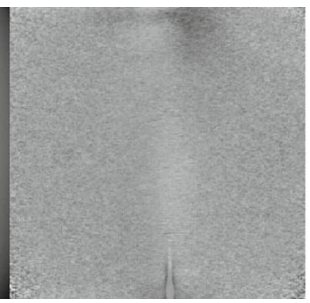

(b)

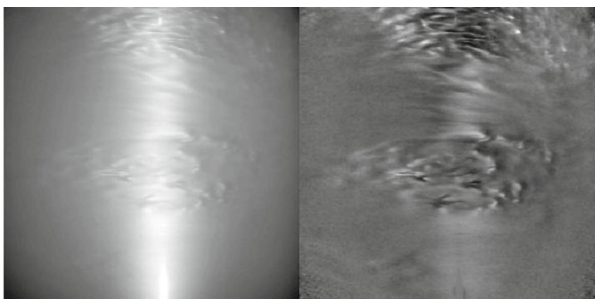

(c) (d)

Fig. 2 Raw (a and c) and normalised (b and d) images of a TC flow at $R e=4530$ with and without a turbulent spot

to platelets in Kalliroscope flakes [21]). In the presence of incident light, areas of varying alignment (caused by the turbulence) reflect different intensities of light as seen in Fig. 2. The fluid was illuminated by exposing the cylinders to white halogen light from a projector. Straight illumination of the curved geometry led to a specular reflection. Therefore, an angle of 45 degrees between the projector and the axis of the cylinders was chosen (as depicted in Fig. 1). A CCD camera with a frame rate of $10 \mathrm{~Hz}$ and a resolution of $500 \times 2800$ pixels (corresponding to $37.5 \mathrm{~mm}$ width $\times 200 \mathrm{~mm}$ height in physical space) was used to capture the reflection.

Each image, defined as a matrix $I$, was subtracted and normalised by the average of one hundred images taken from the laminar flow, $I_{a v g}$, in order to obtain a filtered image, $I_{f i l}=\left(I-I_{a v g}\right) / I_{a v g}$. Among different properties of the filtered image, standard deviation $\sigma$ was found to be the most evident indicator for the spot existence. This is shown in Fig. 3 where the disappearance of a spot results in a considerable drop in the signal amplitude at $t=205 \mathrm{sec} . \sigma$ was calculated for each image and compared with a threshold obtained from $\sigma$ of a fully turbulent flow. If it remained smaller than the threshold for 40 consecutive images (corresponding to $4 \mathrm{sec}$ ), the last image with $\sigma$ higher than the threshold was chosen

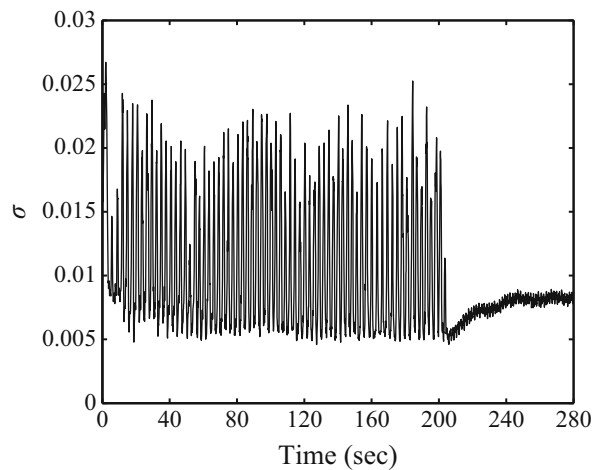

(a)

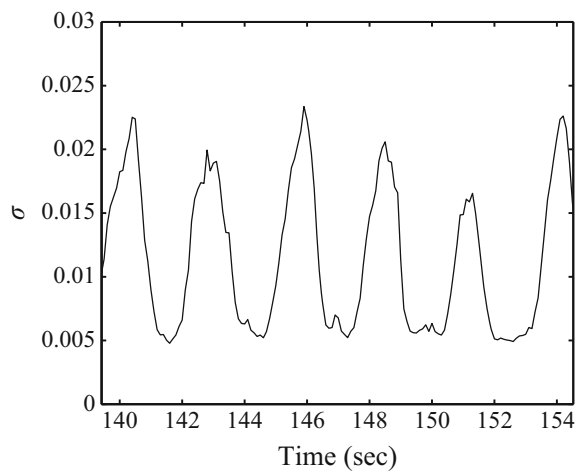

(b)

Fig. 3 a Time series of $\sigma$ (the spot is created by GP at $R e=4530$ ). $\mathbf{b}$ The zoom-in view of the $\sigma$ signal where peaks and troughs correspond to the images with and without the spot. The distance between two peaks indicates the time that the spot completes a full round 


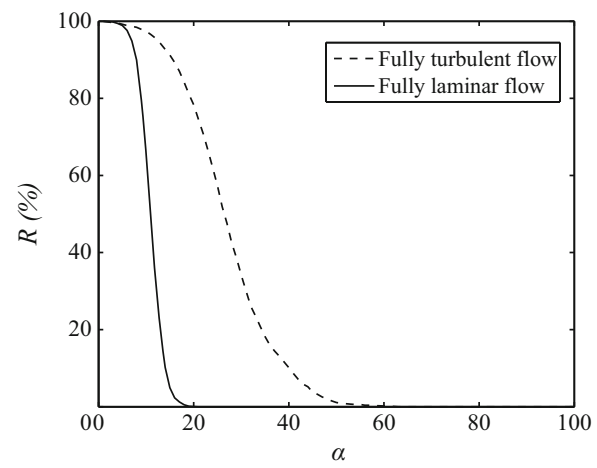

(a)

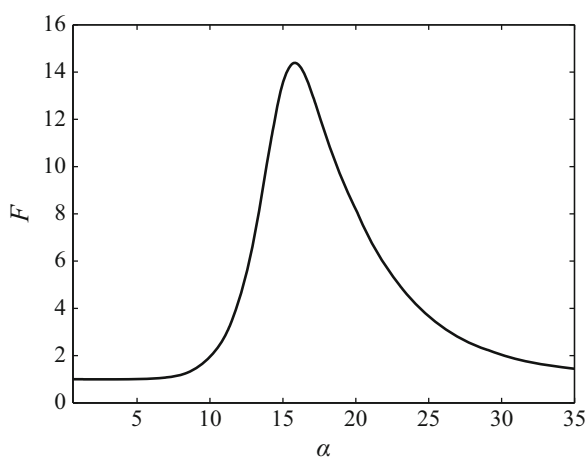

(b)

Fig. 4 Threshold to distinguish laminar and turbulent regions. a Filtered image matrix of a fully laminar and a turbulent flow and $\mathbf{b}$ Signal-to-noise ratio as a function of $\alpha$

as the final spot location. The $4 \mathrm{sec}$ waiting period was chosen to ensure that even at the lowest $R e$, a spot had sufficient time to complete a full round. The accuracy of this approach was visually verified with a stopwatch at random.

The size of spots was calculated as follow: in order to avoid dark corners, a narrow strip from the centre of an image matrix was cut out, $I_{\text {strip }}$, and then filtered:

$$
\alpha=\left|\frac{I_{\text {strip }}-I_{\text {avg }}^{\text {strip }}}{I_{\text {avg }}^{\text {strip }}}\right| \times 100 .
$$

Pixels with $\alpha \geq 16$ were specified as turbulent regions while the rest as the laminar background. This threshold was found to be the optimum point for the signal-to-noise ratio function $F$ defined as:

$$
F(\alpha)=\frac{\left(100-R_{L}\right)+R_{T}}{\left(100-R_{T}\right)+R_{L}},
$$

where $R_{L}$ and $R_{T}$ are the percentages of the laminar and turbulent pixels in a fully laminar and a fully turbulent flow respectively (as displayed in Fig. 4). Finally, consecutive strips were attached to each other and the spot was fully reconstructed.

\section{Results and Discussions}

\subsection{Spot lifetime}

In order to create a spot, GP was applied. During GP, turbulence occupied the whole geometry (as shown in Fig. 5a). Immediately after stopping the inner cylinder, the turbulence was collapsed into a narrow ring below the top plate (Fig. 5b). From then on, depending on unmeasured circumstances, one of the following scenarios occurred: either the turbulent ring vanished within almost $18 \mathrm{sec}$ and the flow became completely laminar, or it transformed to a spot with a tilted shape mostly extended up to the top plate. The spot rotated with the flow for some time, and then suddenly disappeared without any precursor. 


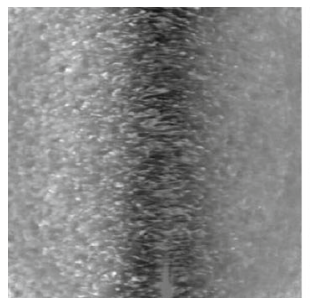

(a)

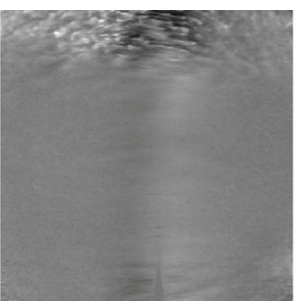

(b)

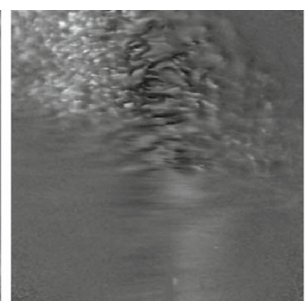

(c)

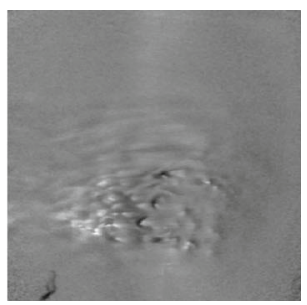

(d)

Fig. 5 Life cycle of a spot at $R e=4530$ with GP. Images (a), (b), (c), and (d) are taken at $t=2, t=14$, $t=52$, and $t=129 \mathrm{sec}$, respectively. Image (d) shows the spot in its last round before decay

The lifetime experiment was repeated 100 times for each Reynolds number. ${ }^{3}$ The cumulative probability distribution (that is the probability of the existence of the spot with time) is illustrated in Fig. 6 for several Reynolds numbers where all curves show a salient feature: after an initial time during which the spot forms, the distribution has an exponentially decaying tail. The probability was described as an exponential function of the time and Reynolds number, $P(t, R e) \sim \exp (-(t) / \tau(R e))$, where $\tau$ is the mean characteristic lifetime. This distribution is consistent with those of previous investigations [3-5, 11-13, 17, 23].

It was also observed that the characteristic lifetime grew by increasing the Reynolds number. From various functions suggested by Hof et al. $[12,13]$, it was found that the superexponential function, $\tau(R e)^{-1}=\exp (-\exp (a R e+b))$ with $a=2.01 \times 10^{-3}$ and $b=$ -7.36 , results in the most accurate fit a regression coefficient of 0.98 . This demonstrates that for the studied range the characteristic lifetime increases with Reynolds number faster than exponentially, but does not diverge to infinity.

As mentioned earlier, the spot was mostly attached to the top plate during its lifetime. This might be due to disturbances caused by air bubbles being stuck in the top gap during the filling of the setup, or due to secondary flows causing the spot to drift upwards. The geometry was modified to enhance bubble release and to reduce secondary flows by inserting an extra ring (as depicted in Fig. 1). Hereafter, the setup with and without modifications are referred to as improved design and original design.

In the improved design, the vortex ring mostly formed near the bottom after GP, and the spot randomly travelled over the whole height. Moreover, the characteristic lifetime was considerably decreased due to the removal of unwanted disturbances (either induced by secondary motions or wakes behind the stuck air bubbles). The question remains about the change in the location of the vortex ring from the top to the bottom of the geometry and the possible effects of the spot formation location on the lifetime. To eliminate these effects, the measurement was repeated with the flow being locally perturbed (as explained in Section 2.2). Consequently, no ring was shaped, and the spot was formed at the middle height of the geometry. Figure 7 compares mean characteristic lifetimes measured in different systems. The influence of the perturbation mechanisms on the lifetime is

\footnotetext{
${ }^{3}$ It has to be noted that the observation time in the highest two Reynolds numbers were limited to $20 \mathrm{~min}$. After this time, a large portion of the pigments adhered to the outer cylinder wall, and consequently, distinguishing the spot from the laminar background was not feasible. To check the error introduced by this limitation, the characteristic lifetime, $\tau$, was recalculated for $R e=4530$ with $10 \%$ and $40 \%$ of the data points with higher lifetimes being excluded. In the former case, the resulting characteristic lifetime differed by less than $3.5 \%$ from the one including all data points. This difference reached $10 \%$ in the latter case.
} 


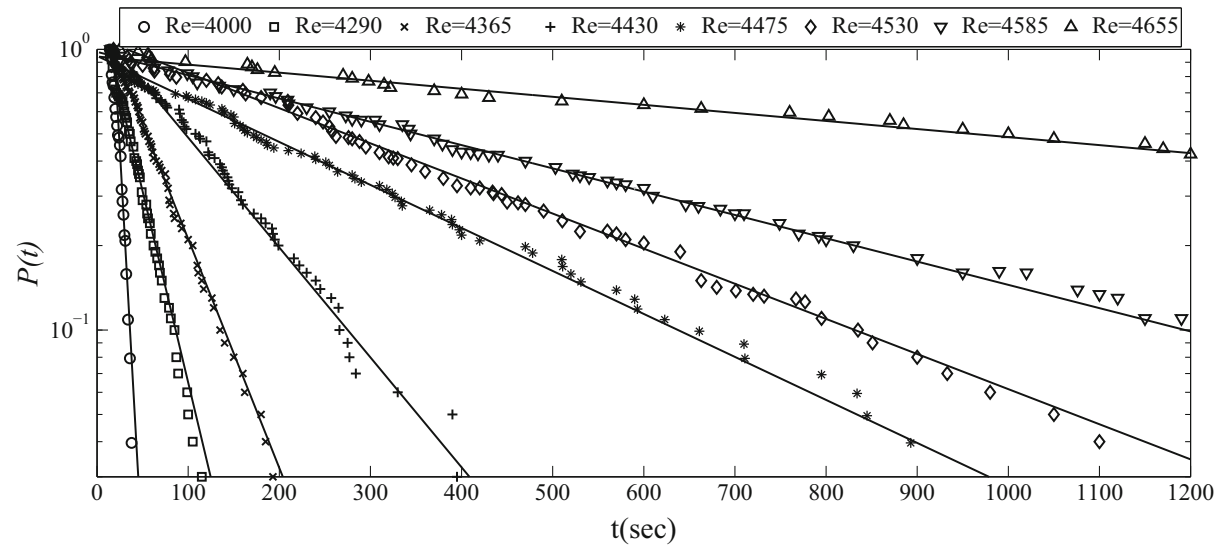

Fig. 6 Probability distributions of lifetimes. Lines are exponential fits through data points which have lifetimes higher than $18 \mathrm{sec}$

inconsequential. This is consistent with previous findings in pipe and plane Couette flows $[3,11-13,17,23]$.

In order to explain the disparity between spot lifetimes in the current and previous study (as displayed in Fig. 7), a scaling approach was suggested. Characteristic lifetimes were scaled based on the geometrical properties ( $\Gamma$ and $\eta$ ) and $R e_{c r}$ of the setups. Advection time unit, $t_{a}=d / r_{o} \Omega_{o}$, was identified as another relevant scaling parameter since it translates the total lifetime period to the total number of rotations. By choosing these parameters, the scaled results were coincided as shown in Fig. 8. It is noteworthy that $R e_{c r}$ itself is dependent on $\eta$ (Dubrulle et al. [9]). As suggested by Richard and Zahn [19], $R e_{c r}$ can be described as $R e_{c r}=1400+550000(1-\eta)^{2}$. Therefore, the spot decay is a function of $R e$, $\Gamma$, and $\eta$ whereas in pipe and plane Couette flows it only depends on $R e$.

\subsection{Spot size}

As opposed to pipe flows where puffs have a fixed size for $R e<R e_{c r}$ [16], the spot size fluctuated during its lifetime (Fig. 9); it shrank, expanded, and splitted to smaller spots

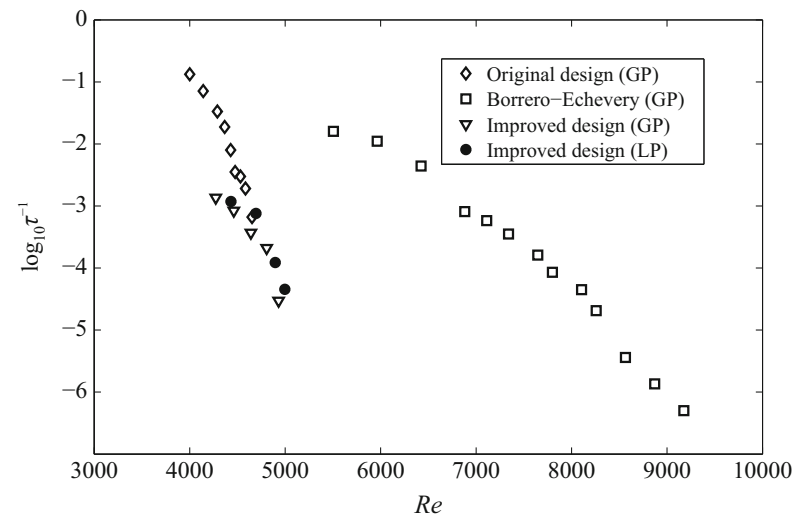

Fig. 7 Characteristic lifetimes in different systems 


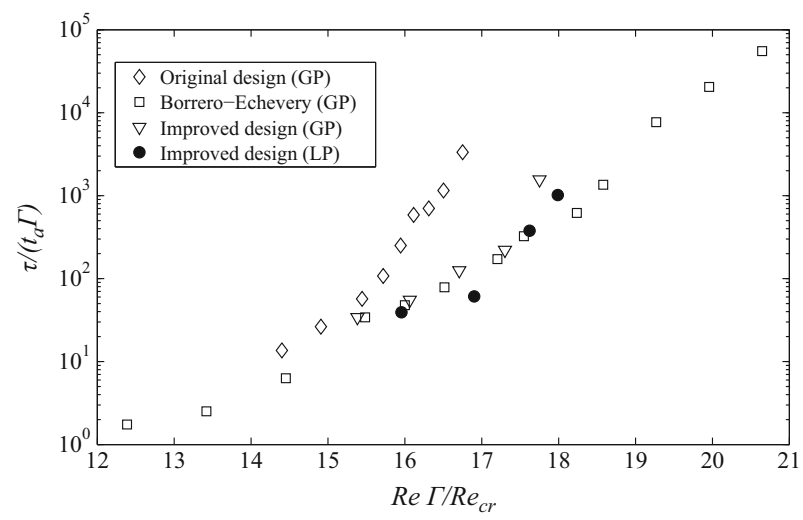

Fig. 8 Scaled characteristic lifetimes

which often merged again. It was observed that the spot had a rather constant width in the streamwise (azimuthal) direction, but its height changed significantly in the spanwise (axial) direction. The average spot size, from generation to disappearance, was used as an indicator to examine the turbulence spread. As $R e$ increased, the average size grew (Fig. 10) and more frequent splitting was observed. At $R e>5000$, the height of the enlarged spot was almost as the height of the cylinder and thus largely impacted by the end boundaries. As discussed in Section 1, to determine the onset of sustained turbulence two factors should be considered: the decay of individual spot and the spatial proliferation of turbulence. Spots had a finite probability of decay as mentioned in previous section. In the studied range of $R e$ (that was limited by the height constraint), no transition from the laminar regime with a localized spot to a spatio-temporally intermittent flow, where probability of proliferation exceeds decay [16], took place. Therefore, no conclusion can be made with respect to the onset of sustained turbulence.
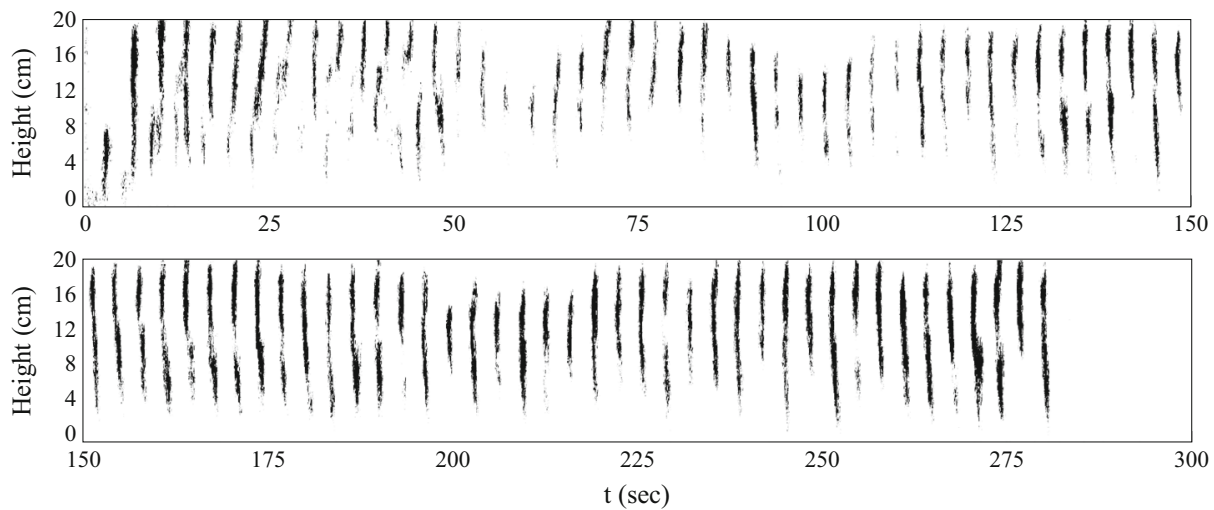

Fig. 9 Spot from generation to disappearance at $R e=4640$. Black points represent turbulent regions whereas white areas are the laminar background 


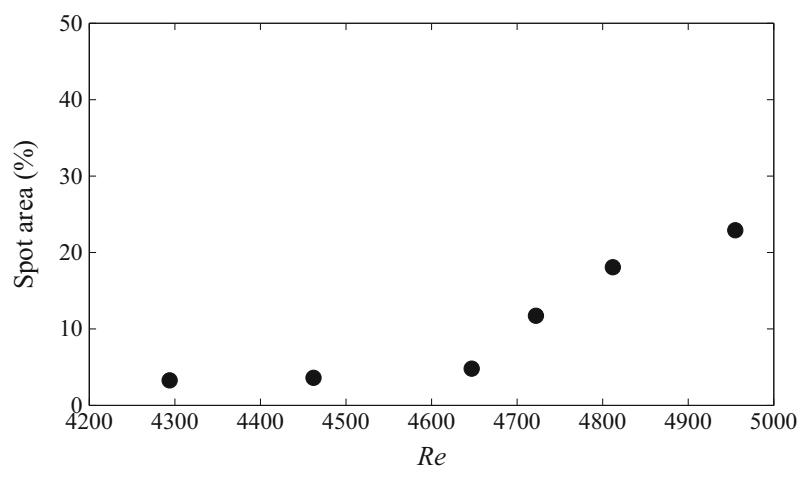

Fig. 10 Percentage of average spot size to total flow area versus Reynolds number

\section{Conclusion}

In the current work, the lifetime of turbulent spots in a Taylor Couette flow with stationary inner cylinder has been studied. Effects of three parameters, namely, boundary conditions, perturbation mechanisms and geometrical properties, on the lifetime were examined. It was shown that the lifetime is considerably impacted by the end boundaries through either introducing unwanted disturbances (e.g. generated by stuck air bubbles) or imposing a secondary flow in the gap. Thus, special attention is required to avoid any adverse geometrical configuration close to the boundaries. In order to eliminate the secondary flow, a setup with stationary end plates should be deployed.

Two perturbation mechanisms (local and global) were tested. Consistent with the results obtained in pipe and plane Couette flows [3,11-13, 17, 23], the lifetime did not depend on the perturbation as long as it was created. A scaling approach was proposed to accommodate for the effects of geometrical properties on the lifetime. It was demonstrated that after scaling, the mean characteristic lifetimes coincided with those of the previous study [3]. Hence, in addition to Reynolds number, the spot decay is a function aspect ratio and radius ratio. This means that the spot decay needs to be described in its scaled form to reveal useful information with respect to the spot temporal evolution. In order to verify the scaling approach, it is suggested to repeat the lifetime measurement in a flexible setup such as the one designed by Avila \& Hof [1] which provides the possibility of testing in several geometrical configurations.

Consistent with the previous findings in pipe and place Couette flows [2, 4, 5, 11, 17, 23], spots had a finite lifetime. The size of a spot varied randomly during its lifetime in contrast with puffs in pipe flows that maintain their size for $R e<R e_{c r}$ [16]. The average spot size increased with $R e$ and reached almost a quarter of the total flow area for $R e \approx 5000$. For the studied range of $R e$ which was limited by the height constraint, no transitional point to a spatio-temporally intermittent flow was observed. To determine the onset of turbulence, where the spread of turbulence is sufficiently faster than the decay of spots, it is suggested to examine the spatio-temporal behavior of spots for a wider range of $R e$ in a setup with a much larger aspect ratio. 
Compliance with Ethical Standards The presented work has been carried out as a part of the author's master's research at Aero \& Hydrodynamics laboratory in Delft University of Technology. Author would like to confirm that there are no potential conflicts of interest (financial or non-financial). Moreover, no human participants or animals were involved in this work.

\section{References}

1. Avila, K., Hof, B.: High-precision Taylor-Couette experiment to study subcritical transitions and the role of boundary conditions and size effects. Rev. Sci. Instrum. 84(6) (2013)

2. Avila, M., Willis, A.P., Hof, B.: On the transient nature of localized pipe flow turbulence. J. Fluid Mech. 646, 127-136 (2010)

3. Borrero-Echeverry, D., Schatz, M.F., Tagg, R.: Transient turbulence in Taylor-Couette flow. Phys. Rev. E. 81(2) (2010)

4. Bottin, S., Chaté, H.: Statistical analysis of the transition to turbulence in plane Couette flow. Eur. Phys. J. B 6(1), 143-155 (1998)

5. Bottin, S., Daviaud, F., Manneville, P., Dauchot, O.: Discontinuous transition to spatiotemporal intermittency in plane Couette flow. EPL (Europhys. Lett.) 43(2), 171 (1998)

6. Carlson, J.A., Jaffe, A., Wiles, A.: The millennium prize problems. Amer. Math Soc. (2006)

7. Chandrasekhar, S.: Hydrodynamic and hydromagnetic stability. Oxford Univ Press, Oxford, UK (1961)

8. Drazin, P.G., Reid, W.H.: Hydrodynamic Stability. Cambridge Univ Press (2004)

9. Dubrulle, B., Dauchot, O., Daviaud, F., Longaretti, P.Y., Richard, D., Zahn, J.P.: Stability and turbulent transport in Taylor-Couette flow from analysis of experimental data. Phys. Fluids 17(9) (2005)

10. Esser, A., Grossmann, S.: Analytic expression for Taylor-Couette stability boundary. Phys. Fluids 8(7), 1814-1819 (1996)

11. Faisst, H., Eckhardt, B.: Sensitive dependence on initial conditions in transition to turbulence in pipe flow. J. Fluid Mech. 504, 343-352 (2004)

12. Hof, B., de Lozar, A., Kuik, D.J., Westerweel, J.: Repeller or attractor? selecting the dynamical model for the onset of turbulence in pipe flow. Phys. Rev. Lett. 101(21) (2008)

13. Hof, B., Westerweel, J., Schneider, T.M., Eckhardt, B.: Finite lifetime of turbulence in shear flows. Nature 443(7107), 59-62 (2006)

14. Koschmieder, E.L.: Bénard cells and Taylor vortices. Cambridge Univ Press (1993)

15. Meseguer, Á., Trefethen, L.: Linearized pipe flow to Reynolds number $10^{7}$. J. Comput. Phys 186(1), 178-197 (2003)

16. Moxey, D., Barkley, D.: Distinct large-scale turbulent-laminar states in transitional pipe flow. P. Natl. Acad. Sci. USA 107(18), 8091-8096 (2010)

17. Peixinho, J., Mullin, T.: Decay of turbulence in pipe flow. Phys. Rev. Lett 96(9) (2006)

18. Reynolds, O.: An experimental investigation of the circumstances which determine whether the motion of water shall be direct or sinuous, and of the law of resistance in parallel channels. Proc. R. Soc. Lond 35, 84-99 (1883)

19. Richard, D., Zahn, J.P.: Turbulence in differentially rotating flows. Astron. Astrophys 347(734) (1999)

20. Romanov, V.: Stability of plane-parallel Couette flow. Funct. Anal. Appl. 7(2), 137-146 (1973)

21. Savas, Ö.: On flow visualization using reflective flakes. J. Fluid Mech 152, 235-248 (1985)

22. Taylor, G.I.: Stability of a viscous liquid contained between two rotating cylinders. Phil. Trans. Roy. Soc. Lond 223, 289-343 (1923)

23. Willis, A.P., Kerswell, R.R.: Critical behavior in the relaminarization of localized turbulence in pipe flow. Phys. Rev. Lett 98(1) (2007)

24. Wygnanski, I.J., Sokolov, M., Friedman, D.: On transition in a pipe. part 2. the equilibrium puff. J. Fluid Mech. 69(02), 283-96304 (1975) 